\title{
Ocorrência e comportamento sociodemográfico de pacientes com leishmaniose tegumentar americana em Vicência, Pernambuco, no período de 2007 a 2014
}

\section{Occurrence and sociodemographic behavior of American cutaneous leishmaniasis in Vicência, Pernambuco, in the period from 2007 to 2014}

\author{
Patrícia Pereira Vasconcelos ${ }^{1}$, Natália Jerônimo de Araújo ${ }^{2}$, Francisca Janaína Soares Rocha ${ }^{3}$
}

\section{Resumo}

A leishmaniose é uma doença negligenciada que está entre as cinco doenças infecto-parasitárias endêmicas de maior relevância. O trabalho teve por objetivo analisar a frequência e o perfil epidemiológico e sociodemográfico dos pacientes com Leishmaniose Tegumentar Americana e acompanhar as medidas de controle. Trata-se de um estudo quantitativo de caráter retrospectivo. Do total dos pacientes acometidos, verificou-se que 59,4\% deles eram do sexo masculino, $33,5 \%$ possuíam idade menor do que 12 anos, $83,1 \%$ eram pardos e 79,3\% estudaram até o ensino fundamental incompleto. Observou-se que a maioria dos diagnósticos para leishmaniose tegumentar americana é emitido baseando-se apenas pelo critério clínico epidemiológico, tendo em vista que os exames parasitológicos e imunológicos foram raramente utilizados para diagnóstico. O maior foco da doença concentrou-se nas áreas de abrangência da Unidade da Saúde da Família (USF) do Imbu, correspondendo a 79,3\% dos casos. Em relação às medidas de controle, observou-se que $69,2 \%$ dos profissionais possuíam conhecimentos sobre leishmaniose, $84,6 \%$ das unidades de saúde da família não desenvolviam programas de ação e estratégias, 76,9\% dos profissionais não participaram de atividades educativas na comunidade, porém, há divulgação à população sobre a ocorrência dos casos de LTA $(69,2 \%)$. Apesar da alta frequência da ocorrência de leishmaniose tegumentar americana no município, as USFs não estavam atuantes no que concernem as medidas de controle referente ao agravo e não participavam de programas de educação em saúde.

Palavras chave: Epidemiologia. Saúde Pública. Diagnóstico. Leishmaniose cutânea.

\begin{abstract}
Leishmaniasis is a neglected disease that is among the top five endemic infectious and parasitic diseases of major relevance. The aim of this study was to analyze the frequency, the demographic, and epidemiological profile of the patients with tegumentary leishmaniasis Americana and accompanying measures of control. Method: It is a retrospective and quantitative study. It was verified that $59.4 \%$ of affected patients were male, $33.5 \%$ were younger than 12 years, $83.1 \%$ were brown and $79.3 \%$ studied until the elementary school. It was observed that the most diagnostic of American cutaneous leishmaniasis were given only by relying on epidemiological clinical judgment, whereas parasitologic and immunologic tests were rarely used for diagnosis. The largest outbreak of the disease was concentrated in the areas of family health unit (USF) the Imbu, corresponding to $79.3 \%$ of the cases. Regarding control measures, it was observed that $69.2 \%$ of professionals knew about leishmaniasis, $84.6 \%$ of family health units did not develop action programs and strategies, $76.9 \%$ of professionals did not participate in educational activities in the community, but there is
\end{abstract}

\footnotetext{
${ }^{1}$ Graduação em Enfermagem pela Universidade Federal de Pernambuco. Enfermeira Residente pelo Instituto de Medicina Integral Professor Fernando Figueira; Recife, Pernambuco, Brasil. E-mail: patricya_vasconcelos@hotmail.com

${ }^{2}$ Especialização em Emergência e UTI pela Universidade Federal de Pernambuco. Enfermeira da Atenção Básica pela Prefeitura Municipal de Saúde de Vicência; Pernambuco, Brasil.

${ }^{3}$ Doutorado em Human Biology - Universitat Erlangen-Nurnberg (Friedrich-Alexander), Alemanha. Docente da Universidade Federal de Pernambuco, Centro de Ciências da Saúde, Departamento de Medicina Tropical; Recife, Pernambuco, Brasil.
} 
disclosure to the public about the occurrence of American cutaneous leishmaniasis cases (69.2\%). Despite the high frequency of LTA in the city, the USFs were not active in that concern the control measures relating to the problem and did not participate in health education programs.

Keyword: Epidemiology. Public Health. Diagnosis. Cutaneous leishmaniasis.

\section{Introdução}

A leishmaniose é considerada uma doença negligenciada que afeta milhares de pessoas ao redor do mundo e atinge, principalmente, pessoas de nível socioeconômico baixo (ROMÃO et al., 2007). Além disso, a leishmaniose é um problema de saúde pública mundial e ressaltada pela Organização Mundial da Saúde (OMS) como uma das cinco doenças infectoparasitárias endêmicas de maior relevância (GUERRA et al., 2006). Apresenta-se sob duas formas de manifestação: a leishmaniose visceral americana (LVA), uma das formas mais severas da doença, conhecida também como Calazar, e a leishmaniose tegumentar americana (LTA).

Estima-se que os casos de leishmaniose atinjam no mundo um total de 1,6 milhões de pessoas anualmente. Destes, 500 mil são de LVA e 1,1 milhão é de LTA. A prevalência total indica que 12 milhões de pessoas em 88 países sofram de leishmaniose e 350 milhões de pessoas vivam em áreas de risco (MIRANDA et al., 2011).

No Brasil, entre o período de 1993 a 2012, a LTA apresentou uma média anual de 26.965 casos autóctones registrados, sendo que o coeficiente médio de detecção foi de 15,7 casos/100.000 hab. A região Norte do Brasil contribuiu com o maior número de casos, correspondendo a aproximadamente $37,3 \%$ do total de casos registrados. Os coeficientes médios mais elevados foram os da região Norte $(73,3$ casos $/ 100.000$ hab.), Centro-Oeste (35,4 casos/100.000 hab.) e Nordeste (18,8 casos/100.000 hab.) (BRASIL, 2014).

A LTA é uma doença infecciosa, não contagiosa, causada por diferentes espécies de protozoários do gênero Leishmania, sendo que no Brasil, a $L$. braziliensis é a espécie que tem maior abrangência e é o principal causador da doença (CUNHA; LIMA; POMPEU, 2006). É uma zoonose que afeta inicialmente animais e secundariamente o homem, tendo no último o período médio de incubação de dois meses (MIRANDA et al., 2011). O modo de transmissão consiste da picada do inseto vetor denominado flebotomíneo, que constitui um grupo de insetos hematófagos conhecidos popularmente como mosquitos-palha e biriguis (BRASIL, 2010). Vale ressaltar que a transmissão ocorre em áreas que circulam o vetor, nas últimas décadas, têm-se sugerido mudanças no padrão de transmissão da LTA, inicialmente a doença era considerada zoonose que acometia ocasionalmente pessoas em contato com as florestas, atualmente a doença começou a ocorrer em zonas rurais, já praticamente desmatadas, e em regiões periurbanas por provável mudança nos hábitos do mosquito vetor (BRASIL, 2010).

O amplo espectro de manifestações clínicas da doença é devido à diversidade de espécies envolvidas com a LTA e do estado e resposta imunológica do indivíduo infectado (BRASIL, 2010). Desse modo, pode apresenta-se na forma cutânea caracterizada por úlceras únicas ou múltiplas, que podem cicatrizar espontaneamente. Todavia, algumas vezes, pode manifestar-se a forma cutaneomucosa com lesões cutâneas que afetam mucosa nasal, oral, faringe e laringe, levando $5 \%$ destes pacientes a óbito por complicações (SAMPAIO; PAULA, 1999). Uma forma clínica rara, porém grave, é a forma cutânea difusa, que ocorre em pacientes com anergia e deficiência específica na resposta imune celular a antígenos de Leishmania, caracteriza-se por lesões nodulares não ulceradas, precedidas por úlcera única que evolui com disseminação linfática do parasito (MURBACK et al., 2011).

Por tais motivos, a LTA é umas das afecções dermatológicas que merece mais atenção devido à sua magnitude, tanto pelo risco de ocorrência de deformidades que pode produzir no ser humano, quanto pelo envolvimento psicológico, com reflexos no desempenho social e econômico dos indivíduos acometidos (BRASIL, 2010).

Atualmente, a LTA é encontrada em todos os estados brasileiros sob distintos perfis epidemiológicos e padrões de transmissão decorrente às modificações socioambientais, e a incidência da doença no Brasil tem aumentado nos últimos 20 anos atingindo indivíduos de diferentes faixas etárias e em ambos os sexos (SILVA; LARROTE; GALATI, 2010). Conforme dados do Ministério da Saúde, no período 
de 1990 a 2008, o Brasil apresentou, em média, 27.608 casos de LTA notificados por ano (OLIART-GUZMÁN et al., 2013). A Região Nordeste corresponde por 39\% dos casos notificados de LTA do Brasil (PADILHA; PEDROSA; ALBUQUERQUE, 2010).

Considerando a abrangência da LTA e os problemas encontrados para seu tratamento, faz-se necessário o estabelecimento de medidas eficazes de profilaxia e de controle da doença e uma saúde pública atuante na comunidade. As medidas gerais de controle da LTA envolvem a vigilância epidemiológica; medidas de atuação na cadeia de transmissão, onde as estratégias de controle devem ser flexíveis e distintas adequadas a cada região ou foco particular, medidas de proteção individual, educativas e administrativas (BRASIL, 2010).

As Secretarias de Vigilância em Saúde orientam que ações voltadas para o diagnóstico precoce e o tratamento adequado dos casos de LTA sejam de responsabilidade das Secretárias Municipais de Saúde, com o apoio das Secretárias Estadual de Saúde e do Ministério da Saúde, que devem organizar uma rede básica de saúde para realizar investigação, assistência e acompanhamento dos casos de leishmaniose, e quando necessário, encaminhar os pacientes com suspeita de LTA para as unidades de referência ambulatorial ou hospitalar. Além disso, devem ser oferecidas condições para a realização do diagnóstico e tratamento precoces (BRASIL, 2014).

A busca ativa de casos na área de foco, ações dos agentes comunitários de saúde e atuação da enfermagem são medidas eficientes para diagnosticar e tratar precocemente os casos detectados, além de atividades educativas que possam auxiliar na redução de novos casos.

No Brasil, a LTA é uma doença de notificação compulsória, em que todo caso confirmado deve ser notificado e investigado pelos serviços de saúde, por meio da Ficha de Investigação da Leishmaniose Tegumentar Americana do Sistema de Informação de Agravos de Notificação (SINAN), isso ocasiona grandes benefícios, pois possibilita conhecer os números reais de acometimento dos indivíduos (ROCHA et al., 2015). Além disso, o seu registro é importante para a classificação epidemiológica (caso autóctone ou importado) e o acompanhamento dos casos. Uma vez detectado um caso importado, deverá ser notificado ao serviço de saúde estadual ou municipal do local provável de infecção (BRASIL, 2014). Desse modo, as unidades básicas de saúde têm papel fundamental no diagnóstico, investigação epidemiológica e no preenchimento da ficha de notificação.

No estado de Pernambuco, raramente encontra-se estudos referentes à situação da LTA em seu território. E quando se pensa em estudos voltados para os padrões epidemiológicos da Zona da Mata Pernambucana, observa-se uma escassez. Por isso, é necessário analisar municípios pertencentes a essa região, como, por exemplo, o município de Vicência (PE) que nos últimos anos tem demonstrado um crescente número de casos. O objetivo do estudo foi analisar a prevalência e o perfil epidemiológico e sociodemográfico dos pacientes com LTA no período compreendido entre 2007 e 2014 e avaliar as medidas de controle para os casos no município de Vicência (PE).

\section{Material e Método}

Esse estudo foi realizado nos municípios de Vicência, localizado na Zona da Mata do estado de Pernambuco, Brasil, tendo sido motivado pelo grande número de casos nesta área.

O município de Vicência, fica aproximadamente $87,1 \mathrm{~km}$ de Recife, tem uma extensão de 228,017 $\mathrm{km}^{2}$, com uma população, conforme o último IBGE (2010), de 30.723 habitantes, totalizando 134,74 hab/ $\mathrm{km}^{2}$ e possui dezessete estabelecimentos de saúde distribuídos em seu território.

A pesquisa foi um estudo quantitativo de caráter retrospectivo, e teve como fonte de dados às fichas de notificações dos pacientes com diagnóstico clínico, epidemiológico e laboratorial de LTA, registrados entre 2007 e 2014 pertencentes à base de dados do Sistema Nacional de Agravos de Notificação (SINAN), fornecidos pela Secretaria de Saúde de Vicência.

As variáveis que foram analisadas representam os aspectos diversos da LTA: sociodemográficas (sexo, faixa etária, local de residência, ocupação), clínico-laboratoriais (classificação dos casos, forma clínica, exames laboratoriais) e epidemiológicas (local provável da infecção e a distribuição do número de casos por área de abrangência das Unidades da Saúde da Família- USF).

Para análise dos dados oriundos dos prontuários foi construído um banco de dados no programa EPI INFO, versão 3.5.2, o qual foi exportado para o SPSS onde foi realizada a análise. Para avaliar o perfil dos pacientes, suas variáveis clínicas e epidemiológicas, foram calculadas as frequências percentuais dos mesmos e construídas as respectivas distribuições de frequências. 
Para avaliação do conhecimento e das medidas de controles da LTA foi aplicado um questionário semiestruturado aos profissionais das Unidades de saúde da família (USF). O instrumento foi elaborado pelos próprios pesquisadores com foco nas funções e ações desenvolvidas pelo enfermeiro e demais membros da equipe de saúde na comunidade.

A pesquisa obedeceu aos critérios éticos, sendo aprovado pelo Comitê de Ética em Pesquisa do Centro de Ciências da Saúde da Universidade Federal de Pernambuco, em atendimento à Resolução 466/12 (CAAE n 28898114.1.0000.5208).

\section{Resultados}

Entre o total dos pacientes acometidos (217 casos) pela LTA, observou-se que $59,4 \%$ eram do sexo masculino, $33,5 \%$ possuíam idade menor do que 12 anos, $83,1 \%$ eram pardos, 79,3\% estudaram até o ensino fundamental incompleto e $65,2 \%$ eram estudantes (tabela 1).

Tabela 1 - Perfil dos pacientes acometidos pela Leishmaniose Tegumenar Americana. Vicência (PE), nos anos de 2007 a 2014.

\begin{tabular}{lll}
\hline Fator avaliado & $\mathbf{N}$ & $\mathbf{\%}$ \\
\hline Sexo & 123 & 59,4 \\
Masculino & 84 & 40,6 \\
Feminino & & \\
Idade & 72 & 33,5 \\
$<12$ anos & 40 & 18,6 \\
13 a 18 & 61 & 28,4 \\
19 a 39 & 34 & 15,8 \\
40 a 59 & 8 & 3,7 \\
60 ou mais & & \\
Raça/cor & 29 & 13,6 \\
Branco & 177 & 83,1 \\
Pardo & 7 & 13,3 \\
Preto & & \\
Escolaridade & 16 & 10,7 \\
Analfabeto & 119 & 79,3 \\
Ensino Fundamental incompleto & 10 & 6,7 \\
Ensino Fundamental completo & 4 & 2,7 \\
Ensino Médio incompleto & 1 & 0,7 \\
Ensino Médio completo & & \\
Ocupação & 92 & 65,2 \\
Estudante & 19 & 13,5 \\
Trabalhador rural em geral & 24 & 17,0 \\
Dona de casa & 6,3 \\
Aposentado & Fi & 4,3 \\
\hline
\end{tabular}

Fonte: Sistema Nacional de Agravos de Notificação (2016).
O principal critério diagnóstico utilizado para a confirmação dos pacientes acometidos pela LTA foi o clínico-epidemiológico, correspondendo a 207 casos $(95,4 \%)$, em apenas 10 casos $(4,6 \%)$ foram confirmados com exames laboratoriais apropriados para o diagnóstico de leishmaniose.

Verificou-se que a maioria dos pacientes não realizou o parasitológico direto $(95,4 \%)$, exame que faz o diagnóstico diferencial com outras patologias. A intradermorreação de Montenegro (IRM) também não foi realizada em $99,5 \%$ dos pacientes e o histopatológico, que consiste na procura do parasita em uma biópsia da lesão ulcerada, também não foi realizado em $98,2 \%$.

Tabela 2 - Dados laboratoriais. Vicência (PE), nos anos de 2007 a 2014.

\begin{tabular}{lll}
\hline Fator avaliado & N & \% \\
\hline Parasitológico Direto & & \\
Positivo & $\mathbf{9}$ & 4,1 \\
Negativo & 1 & 0,5 \\
Não realizado & 207 & 95,4 \\
IRM & & \\
Positivo & 1 & 0,5 \\
Não realizado & 216 & 99,5 \\
Histopatológico & & \\
Realizado & 4 & 1,8 \\
Não realizado & 213 & 98,2 \\
\hline
\end{tabular}

Fonte: Sistema Nacional de Agravos de Notificação (2016).

Em relação à distribuição dos fatores epidemiológicos dos casos de LTA observou-se que a maioria dos pacientes foi classificada como caso autóctone $(98,6 \%)$, provavelmente eles foram contaminados no município de residência $(96,8 \%)$ e a doença não foi relacionada ao trabalho $(93,8 \%)$.

A maior parte dos pacientes com LTA no município de Vicência (PE) apresentou a forma cutânea (99,5\%) e a maioria consistia em casos novos $(99,5 \%)$. Apenas um pequeno percentual de pacientes teve manifestação nas mucosas $(0,5 \%)$.

Houve um aumento significativo no número de ocorrência dos casos de LTA no período de 2007 
a 2009. Tal crescimento foi acompanhado por um decréscimo acentuado nos anos posteriores (Figura 1).

Em relação à distribuição dos pacientes avaliados segundo a localização de suas residências, 90,2\%

Figura 1 - Número dos casos de Leishmaniose Tegumentar Americana no município de Vicência (PE), segundo o ano de ocorrência.

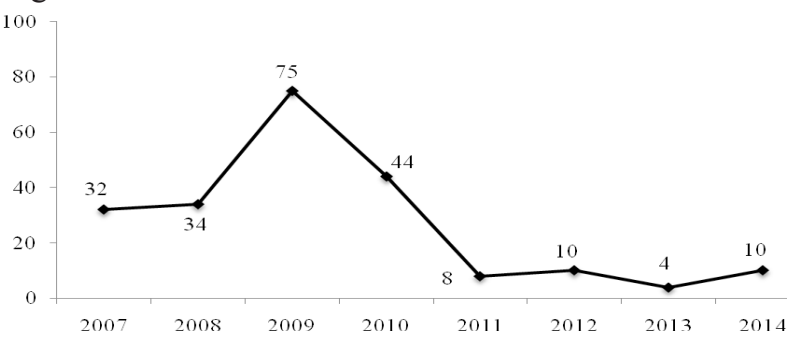

Fonte: Sistema Nacional de Agravos de Notificação (2016).

moravam na área periurbana, 9,3\% na zona urbana e apenas $0,5 \%$ na zona rural.

Em relação à avaliação dos maiores focos de ocorrência de LTA, observou-se que a distribuição dos casos no município concentrou-se nas áreas de abrangência da Unidade da Saúde da Família (USF) do Imbu, correspondendo a 79,3\% dos casos diagnosticados (tabela 3).

Em relação ao acompanhamento e investigação da realização de medidas de controle pelos profissionais

Tabela 3 - Números de casos por área de abrangência da Unidade de Saúde da Família. Vicência (PE), nos anos de 2007 a 2014.

\begin{tabular}{lll}
\hline Área de abrangência & $\mathbf{N}$ & $\mathbf{\%}$ \\
\hline USF Imbu & 172 & 79,3 \\
USF Comunidade Cristã & 10 & 4,6 \\
USF Angélicas & 10 & 4,6 \\
USF Murupé & 8 & 3,7 \\
USF Usina Barra & 5 & 2,3 \\
USF JRMC Figueiredo & 4 & 1,8 \\
Demais unidades (5) & 8 & 3,7 \\
\hline
\end{tabular}

Fonte: Sistema Nacional de Agravos de Notificação (2016). da equipe de saúde das USFs, observou-se que 69,2\% dos profissionais possuíam conhecimentos sobre a leishmaniose, $84,6 \%$ das unidades não tinham programas de ação estratégias para combate da LTA, $76,9 \%$ dos profissionais não participavam de atividades educativas na comunidade. Todavia, havia divulgação à população sobre a ocorrência dos casos de LTA $(69,2 \%)$ e acompanhamentos dos casos acometidos que estavam em tratamento (100\%).

Apesar do grande número de casos de leishmaniose no município de Vicência, percebe-se que os

Tabela 4 - Ações e estratégias de medidas de controle realizadas pelos profissionais de saúde das unidades de saúde de maior foco de LTA, Vicência (PE), 2014.

\begin{tabular}{lccc}
\hline Ações e estratégias & Não & Sim & $\begin{array}{c}\text { Não sabe } \\
\text { informar } \\
\text { \%o }\end{array}$ \\
\hline $\begin{array}{l}\text { Conhecimento de LTA de } \\
\text { um modo geral }\end{array}$ & 30,8 & 69,2 & 0,0 \\
$\begin{array}{l}\text { Programa de ação } \\
\text { estratégica para combate } \\
\text { do agravo no serviço de }\end{array}$ & 84,6 & 15,4 & 0,0 \\
$\begin{array}{l}\text { saúde } \\
\text { Participa das atividades } \\
\text { educativas a respeito das } \\
\text { principais orientações } \\
\text { preventivas da LTA }\end{array}$ & 76,9 & 23,1 & 0,0 \\
$\begin{array}{l}\text { Divulgação à população } \\
\text { sobre a ocorrência dos } \\
\text { casos de LTA }\end{array}$ & 30,8 & 69,2 & 0,0 \\
$\begin{array}{l}\text { Acompanhamentos dos } \\
\text { pacientes acometidos } \\
\text { pelo agravo }\end{array}$ & & & \\
$\begin{array}{l}\text { Orientação das equipes } \\
\text { do programa de agentes } \\
\text { comunitário de saúde } \\
\text { para detecção dos casos } \\
\text { suspeitos }\end{array}$ & 46,1 & 46,1 & 7,8 \\
$\begin{array}{l}\text { Existência de captura } \\
\text { entomológica ou manual } \\
\text { em locais possíveis de } \\
\text { criação e repouso dos } \\
\text { flebotomíneos }\end{array}$ & 69,2 & 15,4 & 15,4 \\
\hline Questionario respondido pelo & 0,0 \\
& & & \\
\hline
\end{tabular}

*Questionário respondido pelos profissionais de saúde de Vicência (PE).

Fonte: Dos Autores. 
profissionais de saúde pertencentes as Unidade de Saúde da Família de maior concentração de LTA não realizavam ampla orientação aos agentes comunitários de saúde (ACS) para detecção precoce dos casos suspeitos dessa enfermidade $(46,1 \%)$, como também $69,2 \%$ dos profissionais afirmaram que não havia captura entomológica ou manual dos flebotomíneos para saber a principal espécie envolvida na ocorrência do agravo (Tabela 4).

\section{Discussão}

A LTA ocorre em ambos os sexos e em todas as faixas etárias, porém, estudos apontam que a média do país predomina nos maiores de 10 anos, representando $90 \%$ dos casos e o sexo masculino, com $74 \%$ (BRASIL, 2010). O que também foi observado anteriormente por Silveira (1999) e Monteiro (2008) no Estado do Paraná.

A LTA é considerada uma doença ocupacional, porque na maioria dos casos a exposição é tipicamente relacionada às atividades laborais e tem como consequência o sofrimento psicológico induzido pelo fato da doença refletir no desempenho social e econômico dos indivíduos acometidos (MIRANDA et al., 2011).

Comparando com estudos nacionais, nosso estudo apresentou resultados distintos, visto que outros estudos mostram que a maioria dos casos de LTA ocorreu em pacientes jovens do sexo masculino, com predomínio entre 20 e 29 anos, estes que estão na fase produtiva, que se estima de 20 a 50 anos (GUERRA et al., 2007). Todavia, em um estudo realizado no entorno do Parque Nacional dos lençóis maranhenses a doença atingiu todas as faixas etárias estabelecidas, sendo uniformemente distribuídas, com um ligeiro predomínio nos indivíduos adolescentes (10 a 19 anos) (ASSUNÇÃO JÚNIOR et al., 2009).

Observar-se no presente estudo que o diagnóstico da LTA foi, em na maioria, realizado através dos aspectos epidemiológicos e clínicos, não tendo sido realizados os testes parasitológicos recomendados pelo Ministério da Saúde nos pacientes positivos para LTA. O diagnostico clínico-epidemiológico consistiu na história clínica do paciente, dos dados epidemiológicos da área onde o paciente provavelmente foi infectado e da anamnese das lesões observando suas características. Foi possível classificar as formas clínicas da LTA. "A LTA produz um amplo espectro de lesões, o que torna o diagnóstico clínico nem sempre simples ou imediato" (GONTIJO; CARVALHO, 2003).
Vale salientar que, apesar da história clínicoepidemiológica e da presença de lesões típicas de leishmaniose serem consideradas fortes indícios do diagnóstico positivo de LTA, a realização de exames parasitológicos é de extrema importância na sua confirmação, uma vez que essa zoonose faz diagnóstico diferencial com inúmeras doenças que também podem apresentar lesões características semelhantes, como sífilis, tuberculose cutânea, esporotricose, cromomicose, neoplasias cutâneas, úlcera tropical, úlceras de estase venosa entre outras (BRASIL, 2010).

Desta forma, o diagnóstico de certeza da LTA é feito pelo encontro do parasito, ou de seus produtos, nos tecidos ou fluidos biológicos dos hospedeiros (MURBACK, 2011). Portanto, recomenda-se a confirmação do diagnóstico por método parasitológico antes do início do tratamento, principalmente naqueles casos com evolução clínica e lesões atípicas fora do habitual e/ou má resposta a tratamento anterior (BRASIL, 2014).

O decréscimo nos casos foi associado à maior atenção da população decorrente às ocorrências anteriores e à aquisição de conhecimentos sobre a LTA. É importante uma atenção básica atuante na comunidade, que proporcione conhecimentos e orientações sobre a doença. Segundo Uchôa et al. (2004), o conhecimento da população sobre a leishmaniose muitas vezes é restrito nas regiões de sua ocorrência, consequentemente, levando ao retardo na procura do diagnóstico e do tratamento, sendo as populações rurais de área endêmica as mais carentes de informação. Desse modo, na maioria das vezes o conhecimento sobre a enfermidade restringe-se às pessoas que já tiveram a doença ou àquelas que já tiveram casos na família ou vizinhos, ocorrendo desinformação sobre a transmissão e o tratamento, dificultando o estabelecimento de estratégias de controle (CHAGAS et al., 2006; UCHÔA et al., 2004).

As transformações no ambiente e o processo de urbanização crescente acarretam a expansão das áreas endêmicas e o aparecimento de novos focos de leishmaniose, podendo encontrar as espécies de flebotomíneos em novos ambientes, como plantações de monoculturas, no peridomicílio e intradomicílio, facilitando a transmissão da doença (DIAS et al, 2007). As análises epidemiológicas da LTA mostraram que nas últimas décadas houve mudanças no padrão de transmissão da doença, que inicialmente acometia ocasionalmente pessoas em contato com as florestas, e posteriormente começaram a surgir casos em zonas 
rurais, já praticamente desmatadas, e em regiões periurbanas (BRASIL, 2014). Desse modo, observa-se um crescente aumento de casos em áreas periurbanas e peridomicílios.

A prevalência dos casos foi maior nas proximidades da USF do Imbu do que a encontrada nas demais USF. Isso é provável por ser uma área periurbana cercada de cultivo de banana e de matas, ambas propícias para alojamentos dos vetores da LTA, que são insetos conhecidos genericamente por flebotomíneos. Esse resultado foi similar aos estudos realizados por Negrão e Ferreira (2009) em que a proximidade do domicílio às matas foi um fator de risco observado na maioria dos casos estudados

Em um estudo realizado em Manaus, foi observado que o aumento dos números de casos de LTA compreendia entre o final do ano e início do período chuvoso. Isto é justificado pelo aumento da densidade dos vetores nas bases das árvores. Assim, as variações de incidência da leishmaniose poderiam ser influenciadas por fatores geográficos e climáticos, responsáveis pelas flutuações nas populações dos vetores (SILVA; LATORRE; GALATI, 2010).

A enfermagem possui um papel importante, pois é uma profissão que utiliza estratégias como a visita domiciliar, a habilidade de comunicação e o relacionamento interpessoal, para desenvolver ações de promoção da saúde que capacitem o indivíduo e a comunidade a exercerem a reflexão crítica para uma mudança de comportamento comprometida com a saúde (BRAGA; CORTEZ; CARNEIRO, 2011). Partindo desse pressuposto, o enfermeiro pode desenvolver práticas educativas e intervir sobre os determinantes e condicionantes do processo saúdedoença, tais medidas que são articuladas à vigilância epidemiológica e a Estratégia de Saúde da Família.

O controle da LTA deve ser abrangente e abordar alguns aspectos como vigilância epidemiológica, medidas de atuação na cadeia de transmissão e medidas educativas. A investigação epidemiológica na comunidade é um instrumento principal que auxiliará nas formulações de estratégias e ações, e também indicará a necessidade da adoção de medidas de controle da LTA, onde se destaca que o diagnostico precoce e tratamento adequado dos casos humanos, bem como as atividades educativas (BRASIL, 2014).

Em relação às principais orientações para evitar os riscos de transmissão, é importante estimular algumas medidas preventivas individuais e coletivas que são fáceis e acessíveis como: uso de repentes individuais em áreas endêmicas, não se expor às picadas nos horários de atividades do vetor, utilização de mosquiteiros de malha fina e telas nas portas e janelas, limpeza de quintais e terreno, evitar acúmulos de lixos orgânicos, limpeza periódica dos abrigos de animais domésticos entre outras medidas (BRASIL, 2010).

É um ponto positivo a divulgação da comunidade sobre a ocorrência da LTA na região, município, localidade, orientando para o reconhecimento das manifestações clínicas e a procura dos serviços para o diagnóstico e tratamento, quando houver caso suspeito. Além de arcar esforço para a capacitação das equipes dos programas de agentes comunitários de saúde, para a busca ativa dos pacientes e disseminação de orientações e informações.

Para que as ações voltadas ao diagnóstico e ao tratamento precoce sejam hábeis é necessária uma rede básica de saúde organizada para rastrear casos suspeitos, assistir e acompanhar como também encaminhar os pacientes com suspeita de LTA para as unidades de referência ambulatorial ou hospitalar, através do estabelecimento do fluxo de referência e contra-referência.

\section{Conclusões}

O estudo mostrou altos índices de LTA confirmados em Vicência (PE) nos últimos oito anos, prevalecendo em indivíduos do sexo masculino, menores de 12 anos, estudantes e com ensino Fundamental incompleto. Desse modo, a leishmaniose é um agravo que continua ainda acometendo muitos indivíduos e permanecendo um problema de saúde pública.

Este estudo evidenciou alguns problemas relacionados às medidas eficazes de controle da LTA, como a participação dos profissionais em atividades educativas prestadas a população e a ausência de ações e estratégias de controle referente ao agravo.

É de fundamental importância a educação em saúde da população exposta ao risco para o apoio, estimulação de medidas de controle adotadas visando à prevenção de LTA e de outros agravos endêmicos. Vale salientar que apesar do decréscimo de casos nos últimos anos, o controle da patologia deve ser abrangente e abordar alguns aspectos como vigilância epidemiológica, medidas de atuação na cadeia de transmissão e medidas educativas.

As atividades de educação em saúde, as quais exigem mais recursos humanos que materiais, precisam ser inseridas em todos os serviços que promovam ações de vigilância e controle para LTA e para outras 
endemias. O enfermeiro conjuntamente com sua equipe de saúde deve procurar ações e estratégias básicas de disseminação de conhecimentos a população. Assim, poderá ser uma ferramenta auxiliar para a reflexão crítica dos condicionantes de saúde e para a demanda espontânea aos serviços de saúde. Esses fatores são importantes para o diagnóstico e tratamento precoce de casos suspeitos na comunidade.

\section{Referências}

ASSUNÇÃO JÚNIOR, A. N.; SILVA, O.; MORAES, J. L. P.; NASCIMENTO, F. R. F.; PEREIRA, Y. N.; COSTA, J. M. L.; REBÊLO, J. M. Foco emergente de leishmaniose tegumentar no entorno do Parque Nacional dos Lençóis Maranhenses, Nordeste, Brasil. Gazeta Médica da Bahia, Salvador, v. 79, s. 3, p. 103109, 2009.

BRAGA, A. L. S.; CORTEZ, E. A.; CARNEIRO, F. R. Atuação do enfermeiro no controle de endemias. Enfermería Global, Murcia, n. 23, p. 320, 2011.

BRASIL. Ministério da Saúde. Guia de vigilância em saúde. Brasília, 2014.

BRASIL. Ministério da Saúde. Manual de vigilância da leishmaniose tegumentar. Brasília, 2010.

CHAGAS, A. C.; PESSOA, F. A. C.; MEDEIROS, J. F.; PY-DANIEL, V.; MESQUITA, E. C.; BALESTRASSI, D. A. Leishmaniose tegumentar americana (LTA) em uma vila de exploração de minérios - Pitinga, município de Presidente Figueiredo, Amazonas, Brasil. Revista Brasileira de Epidemiologia, Cerqueira César, v. 9, n. 2, p.186-192, 2006.

CUNHA, J. C. L.; LIMA, J. W. O.; POMPEU, M. M. L. Domestic transmission of cutaneous leishmaniasis and the association between human and canine leishmaniasis, during an epidemic in Serra de Baturité, State of Ceará, Brazil. Revista Brasileira de Epidemiologia, Cerqueira César, v. 9, n. 4, p. 425-435, 2006.

DIAS, E. S.; SILVA, J. C. F.; SILVA, J. C.; MONTEIRO, E. M.; PAULA, K. M.; GONÇALVES, C. M.; BARATA, R. A. Flebotomíneos (Diptera: Psychodidae) de um foco de leishmaniose tegumentar no Estado de Minas Gerais. Revista da Sociedade Brasileira de Medicina Tropical, Uberaba, v. 40, n. 1, p. 49-52, 2007.
GONTIJO, B.; CARVALHO, M. L. R. Leishmaniose tegumentar americana. Revista da Sociedade Brasileira de Medicina Tropical, Uberaba, v. 36, n. 1, p. 71-80, 2003.

GUERRA, J. A. O.; BARBOSA, M. G. V.; LOUREIRO, A. C. S. P.; COELHO, C. P.; ROSA, G. G.; COELHO, L. I. A. C. R. Leishmaniose tegumentar americana em crianças: aspectos epidemiológicos de casos atendidos em Manaus, Amazonas, Brasil. Cadernos de Saúde Pública, Rio de Janeiro, v. 23, n. 9, p. 2215-2223, 2007.

GUERRA, J. A. O.; RIBEIRO, J. A. S.; COELHO, L. I. A. R. C.; BARbosA, M. G. V.; PAES, M. G. Epidemiologia da leishmaniose tegumentar na Comunidade São João, Manaus, Amazonas, Brasil. Cadernos de Saúde Pública, Rio de Janeiro, v. 22, n. 11, p. 2319-2327, 2006.

IBGE. Cidades. Vicência, PE. Disponível em: $<$ https:// cidades.ibge.gov.br/>. Acesso em: 1 maio 2016.

MIRANDA, T. M.; MALAQUIAS, L. C. C.; ESCALDA, P. M. F, RAMALHO, K. C.; COURA-VITAL, W.; SILVA, A. R. Estudo descritivo sobre a leishmaniase tegumentar americana na área urbana do Município de Governador Valadares, Estado de Minas Gerais, Brasil. Revista Pan-Amazônica de Saúde, Ananindeua, v. 2, n. 1, p. 27-35, 2011.

MONTEIRO, W. M.; NEITZKE, H. C.; LONARDONI, M. V. C.; SILVEIRA, T. G. V.; FERREIRA, M. E. M. C.; TEODORO, U. Distribuição geográfica e características epidemiológicas da leishmaniose tegumentar americana em áreas de colonização antiga do Estado do Paraná, Sul do Brasil. Cadernos de Saúde Pública, Rio de Janeiro, v. 24, n. 6, p. 1291-1303, 2008.

MURBACK, N. D. N.; FILHO, G. H.; NASCIMENTO, R. A. F.; NAKAZATO, K. R. O.; DORVAL, M. E. M. C. Leishmaniose tegumentar americana: estudo clínico, epidemiológico e laboratorial realizado no Hospital Universitário de Campo Grande, Mato Grosso do Sul, Brasil. Anais Brasileiros de Dermatologia, Rio de Janeiro, v. 86, n. 1, p. 55-63, 2011.

NEGRÃO, G. N.; FERREIRA, M. E. M. C. Leishmaniose tegumentar americana: aspectos geográficos intervenientes na ocorrência da enfermidade no município de Maringá, Paraná. Hygeia: Revista Brasileira de Geografia Médica e da Saúde, Uberlândia, v. 5, n. 8 , p. $115-124,2009$. 
OLIART-GUZMÁN, H.; MARTINS, A. C.; MANTOVANI, S. A. S.; BRAÑA, A. M.; DELFINO, B. M.; PEREIRA, T. M.; SANTOS, A. P.; JÚNIOR, J. A. F.; BRANCO, F. L. C. C.; CAMPOS, R. G.; OLIVEIRA, C. S. M.; MUNIZ, P. T.; NUNES, M. S. Características epidemiológicas da leishmaniose tegumentar americana na fronteira amazônica: estudo retrospectivo em Assis Brasil, Acre. Revista de Patologia Tropical, Goiânia, v. 42, n. 2, p. 187-200, 2013.

PADILHA, B. G.; PEDROSA, F. A.; ALBUQUERQUE, P. V. V. Indicadores epidemiológicos da leishmaniose tegumentar americana, no período de 1999 a 2008, no Estado de Alagoas, Brasil. Revista Pan-Amazônica de Saúde, Ananindeua, v. 1, n. 3, p. 95-102, 2010.

ROCHA, T. J. M.; BARBOSA, A. C. A.; SANTANA, E. P. C.; CALHEIROS, C. M L. Aspectos epidemiológicos dos casos humanos confirmados de leishmaniose tegumentar americana no Estado de Alagoas, Brasil. Revista Pan-Amazônica de Saúde, Ananindeua, v. 6, n. 4, p.49-54, 2015.

ROMÃO, P. R. T.; DIAS, R. O.; CRUZ, K. K.; MARQUES, F. C. S.; MONTEIRO, M. C. Leishmaniose: resposta imune e mecanismos antioxidantes de escape. Revista de Pesquisa e Extensão em Saúde, Criciúma, v. 3, n. 1, p. 1-10, 2007.

SAMPAIO, R. N. R.; PAULA, C. D. R. Leishmaniose tegumentar americana no Distrito Federal. Revista da Sociedade Brasileira de Medicina Tropical, Uberaba, v. 32, n. 5, p. 523-528, 1999.

SILVA, A. F.; LATORRE, M. R. D. O.; GALATI, E. A. B. Fatores relacionados à ocorrência de leishmaniose tegumentar no Vale do Ribeira. Revista da Sociedade Brasileira de Medicina Tropical, Uberaba, v. 43, n. 1, p. 46-51, 2010.

SILVEIRA, T. G. V.; ARRAES, S. M. A.; BERTOLINI, D. A.; TEODORO, U.; LONARDONI, M. V. C. ; ROBERTO, A. C. B. S.; RAMOS, M.; SOBRINHO, A. N.; ISHIKAWA, E.; SHAW, J. Observações sobre o diagnóstico laboratorial e a epidemiologia da leishmaniose tegumentar no Estado do Paraná. Revista da Sociedade Brasileira de Medicina Tropical, Uberaba, v. 32, n. 1, p. 413-423, 1999.

SISTEMA NACIONAL DE AGRAVOS DE NOTIFICAÇÃO - (SINAN). Disponível em: <http:// portalsinan.saude.gov.br/>. Acesso em: 1 maio 2016.
UCHÔA, C. M. A.; SERRA, C. M. B.; MAGALHÃES, C. M.; SILVA, R. M. M.; FIGLIUOLO, L. P.; LEAL, C. A.; MADEIRA, M. F. Educação em saúde: ensinando sobre a leishmaniose tegumentar americana. Cadernos de Saúde Pública, Rio de Janeiro, v. 20, n. 4, p. 935941, 2004.

\section{AGRADECIMENTOS}

À comunidade de Vicência em Pernambuco pela acolhida e a Secretaria Municipal de Saúde pelo fornecimento das fichas de notificação dos pacientes com LTA.

\section{FINANCIAMENTO}

Recursos próprios da equipe executora. 
Vasconcelos, P. P. et al. 